phorylate extracellular lysophospholipids. This modification, which occurs in both somatic and germ cells, is thought to create a lysophospholipid gradient essential for germ cell migration (4).

The work of Kawahara et al. (2) describes the cellular export of a bioactive lipid called sphingosine-1-phosphate (S1P) in zebrafish. It was previously found that S1P activates a $\mathrm{G}$ protein-coupled receptor called Miles Apart (Mil) in zebrafish, and that loss of functional Mil results in the formation of split hearts (cardia bifida) because of the arrested migration of cardiomyocyte precursors. Mil expression in mesodermal cells somehow guides the migration of cardiomyocyte precursor cells toward the midline of the developing embryo, an essential step in the formation of the primitive heart tube (5).

Kawahara et al. conducted a mutagenesis screen in zebrafish and identified a transporter-like protein, Spns2, that acts upstream of Mil. The authors showed that expression of the spns 2 gene in yolk syncytial layer cells is essential for the function of Mil in early heart development. The same gene was identified in another study with a similar mutagenesis strategy (6). The authors further showed that Spns2 protein (or its human or fish orthologs) exports S1P, which then activates Mil in the mesoderm, allowing the proper migration of cardiomyocyte precursors. Moreover, loss of functional Spns2 results in the formation of split hearts, suggesting the essential nature of S1P export in heart development (fig. S1).

In contrast to the Drosophila Mdr49 system, substrate recognition by Spns 2 may be more specific, because the related protein Spns 1 could not transport S1P $(2,6)$. Previous work suggests that $\mathrm{ABCC} 1$ and $\mathrm{ABCA} 1$ transporters (which export sterols, eicosanoids, and other lipophilic molecules) may transport $\mathrm{S} 1 \mathrm{P}$ as well $(7,8)$. However, genetic evidence that these transporters move S1P is lacking, and given the new findings on Spns2, it will be important to assess the role of various transporters in exporting S1P from cells.

Nonetheless, the expression and function of Spns2 may be tightly regulated to establish and/or maintain precise S1P gradients spatially and temporally during embryogenesis. S1P activation of the Mil receptor in the mesodermal cells would then create a permissive environment for the migration of cardiomyocyte precursor cells toward the midline, thereby allowing the formation of the primitive heart tube.

The precise spatial and temporal establishment of lipophilic molecule gradients as guidance cues underlies many diverse biological processes. S1P gradients guide lymphocyte egress from thymus and peripheral lymphoid organs; indeed, modulation of this process is the basis for therapeutic development in autoimmune diseases (9). Further determining how the expression of lipophilic molecules and their transporters is regulated under physiological conditions may reveal whether and how they contribute to pathological processes. Given that transport inhibitors achieve great therapeutic efficacy (as in the case of selective serotonin reuptake inhibitors), better knowledge in this area may contribute to identifying potential drug targets. In addition, geranylgeranylated secreted factors would be inhibited by HMGCR inhibitors (such as statins), which are widely used to control heart disease and stroke. Whether such a mechanism is a part of statins' efficacy remains to be determined.

\section{References}

1. S. Ricardo, R. Lehmann, Science 323, 943 (2009).

2. A. Kawahara et al., Science 323, 524 (2009); published online 11 December 2008 (10.1126/science.1167449).

3. A. C. Santos, R. Lehmann, Dev. Cell 6, 283 (2004).

4. A. D. Renault, Y. J. Sigal, A. J. Morris, R. Lehmann, Science 305, 1963 (2004); published online 26 August 2004 (10.1126/science.1102421).

5. E. Kupperman, S. An, N. Osborne, S. Waldron, D. Y. Stainier, Nature 406, 192 (2000).

6. N. Osborne et al., Curr. Biol. 18, 1882 (2008).

7. P. Mitra et al., Proc. Natl. Acad. Sci. U.S.A. 103, 16394 (2006).

8. K. Sato et al., J. Neurochem. 103, 2610 (2007).

9. S. R. Schwab et al., Science 309, 1735 (2005).

\section{Supporting Online Material}

www.sciencemag.org/cgi/content/full/323/5916/883/DC1 Fig. S1

10.1126/science.1170009

\title{
Making Nice with Viruses
}

\author{
Donald B. Stoltz ${ }^{1}$ and James B. Whitfield ${ }^{2}$
}

$\mathrm{T}$ he polydnaviruses have long been regarded as an anomaly in virology. For example, virus particles, or virions, will readily infect a variety of cell lines, but never replicate in them. The reason is that unlike all other known viruses, that portion of the polydnavirus genome necessary for making progeny virions is in fact not packaged into them (1). On page 926 of this issue, Bézier et al. reveal where the missing genes are: embedded within the chromosomal DNA of the virus's wasp host (2). More importantly, Bézier et al. have uncovered a viral origin for these essential genes - at least in theory, they could have been of wasp origin. In so doing, the authors solve a longstanding mystery, and at the same time estab-

${ }^{1}$ Department of Microbiology and Immunology, Dalhousie University, Halifax, Nova Scotia, B3H 1X5, Canada. E-mail: dstoltz@dal.ca 2Department of Entomology, Urbana, IL 61801, USA. E-mail: jwhitfie@life.uiuc.edu lish a new paradigm in virology: Whereas viruses have been typically seen as either parasites or commensals, we must now recognize a potential for obligatory mutualism.

Beginning in 1967 (3), viruslike particles were being observed in the ovaries of numerous species of parasitic wasps (parasitoids). These entities were present in all females of all affected species, suggesting vertical transmission. It was also evident that the particles were designed for export, initially into oviducts, and

A tale of two tails. An electron micrograph shows similar tail-like appendages (arrows) associated with a bracovirus nucleocapsid (upper left) and a nudivirus capsid (lower right), suggesting a possible phylogenetic relationship between the two types of virus. Unlike nudiviruses, which are conventional viruses, the bracoviruses do not package genes that are necessary for viral morphogenesis. Until now, convincing support for a viral origin of the bracoviruses has remained elusive. Scale bar, $200 \mathrm{~nm}$.
Genomics reveals the origin of a polydnavirus lineage and a new way for viruses and their hosts to live together.

subsequently (along with parasitoid eggs) into the body cavity of the host - usually a caterpillar-being parasitized by the wasp. Viruslike particles from two different parasitic wasp species were eventually isolated and shown to package circular double-stranded DNAs of varying sizes $(4,5)$. This led to the establishment of a new virus family, Polydnaviridae (from polydisperse DNA virus) (6). At least in one case, purified particles

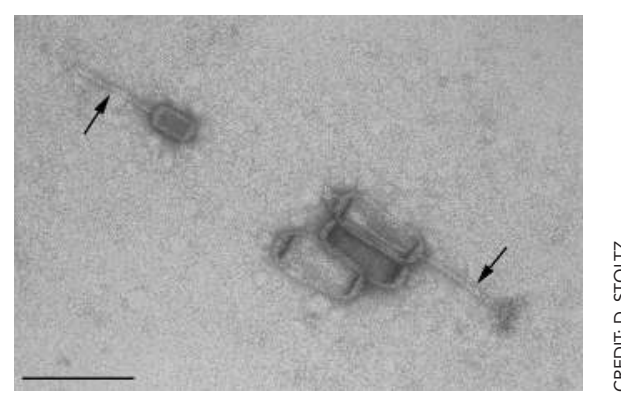


were immunosuppressive, protecting parasitoid eggs from the lethal defensive response that would otherwise be mounted in parasitized caterpillars (7). Because tens of thousands of parasitoid wasp species were probably carrying this type of virus, it was widely assumed that we were looking at mutualism on a grand scale. But, were the polydnaviruses really viruses?

The answer is a matter of semantics, and of how we define a virus. Perhaps the more important issue has to do with origins; that is, are the polydnaviruses a cellular "invention" [a nuclear secretion sensu (8)] or did they originate from conventional viruses? Given that genes encoding viral structural and replicative functions are not packaged into polydnavirions, then they must be sought in wasp genomic DNA. If such genes could be found and shown to have authentic viral relatives, then whatever we decide to call these entities, they must have descended from typical viruses.

As has now been convincingly demonstrated, homologs of genes derived from conventional viruses do indeed reside within wasp chromosomal DNA. Specifically, Bézier et al. have discovered 22 putative nudivirus gene homologs in the wasp genome, including 12 core genes that are also present in a close and well-known relative, the baculoviruses. Interestingly, a putative relationship between bracoviruses (one of the two major recognized polydnavirus lineages) and Oryctes rhinoceros virus was suggested about 30 years ago, on the basis of a remarkable structural resemblance (9) (see the figure). At that time, the Oryctes virus was thought to represent a nonoccluded baculovirus, but recently, it has been assigned to the baculovirus-related genus, Nudivirus (10).

So, why is this important for virology? Again, there's the issue of how to define a virus. Should we exclude the polydnaviruses? Consider a typical definition for "virus": a transmissible agent that in extracellular form consists of a DNA or RNA genome minimally packaged within a protein coat, replicating by the coordinated assembly of subviral components, rather than by growth and division. "Extracellular" implies that all viruses are transmitted horizontally, from one host to another, even if some of them (e.g., temperate bacteriophages) can also on occasion be transmitted vertically within host genomes. In typical definitions of a virus, there is no mention of what the genome must encode, on the assumption that for every virus there is a host that is permissive for productive infection, from which progeny virions will result. This is impossible for polydnaviruses. Much like the recombinant viral vectors used in gene therapy experiments, polydnaviruses are crippled, and so can rightly be viewed as naturally occurring genedelivery vehicles, or even wasp organelles (11). If we do not wish to consider polydnaviruses as bona fide viruses, then the standard definition of a virus must specify that the packaged genome encodes all functions required for viral replication. Finally, it is ironic that in all 1259 pages of the most recent compendium on virus taxonomy (12), "virus" is left undefined.

We would suggest that the more interesting lesson here for virologists and for evolutionary biologists may be that there is now reason to start thinking about virus-host relationships in much broader terms, so as to include not only mutualism, which may be a lot more common than previously contemplated (13, 14), but also obligatory mutualism, as exemplified by the wasp-nudivirus story. How did this kind of relationship arise? Parasitoid larvae feeding within their hosts are exposed to a

variety of viruses and will likely become infected by some. Moreover, the parasitoid ovary may represent a permissive environment for maintaining persistent virus infections (15). It may be that bilateral gene transfer has preserved some elements of these that were of mutual benefit.

\section{References}

1. E. Espagne et al., Science 306, 286 (2004).

2. A. Bézier et al., Science 323, 926 (2009).

3. S. Rotheram, Nature 214, 700 (1967).

4. P. J. Krell, D. B. Stoltz, J. Virol. 29, 1118 (1979).

5. P. J. Krell, D. B. Stoltz, Virology 101, 408 (1980).

6. D. B. Stoltz et al., Intervirology 21, 1 (1984).

7. K. M. Edson et al., Science 211, 582 (1981).

8. W. N. Norton et al., Cell Tissue Res. 162, 195 (1975).

9. D. B. Stoltz, S. B. Vinson, Adv. Virus Res. 24, 125 (1979).

10. Y. Wang et al., Arch. Virol. 152, 519 (2007).

11. B. A. Federici, Y. Bigot, J. Insect Physiol. 49, 419 (2003).

12. C. M. Fauquet et al., Eds., Virus Taxonomy: VIllth Report of the International Committee on Taxonomy of Viruses (Elsevier, London, 2005)

13. J. J. Barondess, J. Beckwith, Nature 346, 871 (1990).

14. F. Arnaud et al., PLoS Pathog. 3, 1716 (2007).

15. D. B. Stoltz, J. B. Whitfield, J. Hym. Res. 1, 125 (1992).

10.1126/science. 1169808

\section{PLANETARY SCIENCE}

\section{Seeing the Missing Half}

\section{Gregory A. Neumann' and Erwan Mazarico ${ }^{1,2}$}

Results from the Japanese SELENE mission shed light on differences between the far and nearsides of the Moon.

$\mathrm{T}$ he Moon is our closest planetary neighbor and the only extraterrestrial body to which humans have traveled, yet many questions about its origin and early history remain unanswered. Four papers published in this issue by scientific teams of the Japanese SELENE (Kaguya) mission (1-4) offer a new global view of the Moon that helps to elucidate how the Moon evolved to its present state.

The Moon is lopsided: Its visible nearside (tidally locked to face the Earth) is covered with smooth, dark volcanic mare, whereas the farside mainly consists of more heavily cratered, bright highland material. The differences in crustal thickness and density, apparent surface age, composition, and volcanic activity between the two sides are variously ascribed to external causes (such as a giant impact) or to internal causes (such as core formation, mantle convection, and crustal differentiation). The key to resolving these questions will be better data.

${ }^{1}$ NASA Goddard Space Flight Center, Code 698, Greenbelt, MD 20771, USA. 2 NASA Postdoctoral Program Fellow, Goddard Space Flight Center, Greenbelt, MD 20771 USA. E-mail: gregory.a.neumann@nasa.gov; erwan.m. mazarico@nasa.gov
The Apollo missions that ended in 1972 led to the current paradigm for the Moon's formation following a collision between the early Earth and a Mars-sized body (5). Analysis of lunar samples led to the hypothesis that the Moon was initially engulfed in a deep magma ocean and then differentiated to form a crust different from that of Earth. This crust subsequently hardened but was battered by meteorites during the late heavy bombardment that ended around 3.8 billion years ago, resulting in a surface covered by basins.

Some basins are as large as $2500 \mathrm{~km}$ across and $13 \mathrm{~km}$ deep (6), unlike anything on Earth. Their preservation indicates that the Moon's lithosphere formed rapidly and has since remained intact, but gives few clues to the present structure and thermal state of the lunar interior. The Apollo seismometers resolved the shallow crustal structure in a few locations, and detected deeper moonquakes whose origin remains elusive. A core-mantle boundary has not been detected, but data from more than 30 years of laser ranging to retroreflectors left on the lunar surface are consistent with a small liquid core (7). 